\title{
Psychosocial outcome and psychiatric comorbidity in older adolescents with Tourette syndrome: controlled study ${ }^{\dagger}$
}

Daniel A. Gorman, Nancy Thompson, Kerstin J. Plessen, Mary M. Robertson, James F. Leckman and Bradley S. Peterson

\section{Background}

Children with Tourette syndrome generally experience improvement of tics by age 18 years, but psychosocial and comorbidity outcomes at this age are unclear.

\begin{abstract}
Aims
To compare psychosocial outcomes and lifetime comorbidity rates in older adolescents with Tourette syndrome and controls. We hypothesised a priori that individuals with Tourette syndrome would have lower Children's Global Assessment Scale (CGAS) scores.
\end{abstract}

\section{Method}

A total of 65 individuals with Tourette syndrome, identified in childhood, and 65 matched community controls without tic or obsessive-compulsive disorder (OCD) symptoms were assessed around 18 years of age regarding psychosocial functioning and lifetime psychiatric disorders.

\section{Results}

Compared with controls, individuals with Tourette syndrome had substantially lower CGAS scores $\left(P=10^{-8}\right)$ and higher rates of attention-deficit hyperactivity disorder (ADHD), major depression, learning disorder and conduct disorder $(P \leqslant 0.01)$ In the participants with Tourette syndrome, poorer psychosocial outcomes were associated with greater ADHD, OCD and tic severity.

\section{Conclusions}

Clinically ascertained children with Tourette syndrome typically have impaired psychosocial functioning and high comorbidity rates in late adolescence.

\section{Declaration of interest}

None.
Tourette syndrome is a childhood-onset neuropsychiatric disorder defined by motor and vocal tics that persist for $>1$ year. In addition to having tics, children with Tourette syndrome often function poorly across numerous psychosocial domains ${ }^{1}$ and have high rates of psychiatric comorbidity. ${ }^{2}$ The long-term course of Tourette syndrome is variable, but tics typically improve during adolescence such that by age 18 years, up to $90 \%$ of individuals with Tourette syndrome have only mild tics or none at all. ${ }^{3,4}$ It is unclear, however, whether their psychosocial functioning, like their tics, improves by this age. Previous longitudinal studies found that individuals with Tourette syndrome who were followed up in late adolescence or early adulthood functioned reasonably well, but these studies were limited by the absence of a control group. ${ }^{5-7}$ In contrast, controlled cross-sectional studies reported that adults with Tourette syndrome had poorer quality of life ${ }^{8}$ and more psychopathology, ${ }^{9}$ but a critical limitation was that the participants were not necessarily identified as having Tourette syndrome in childhood and were attending specialty clinics as adults. Consequently, the poor outcomes described may apply mainly to the minority of individuals with Tourette syndrome who continue to have prominent tics and pursue treatment for them in adulthood. Our objective, therefore, was to reassess in late adolescence a large sample of individuals who were identified as having Tourette syndrome in childhood and may or may not still be in treatment, and compare them with community controls with respect to psychosocial functioning and psychiatric illness. We selected a community control group because we wanted to address

TPortions of this manuscript were presented in a symposium and as a poster at the 53rd Annual Meeting of the American Academy of Child and Adolescent Psychiatry, Boston, MA, 23-28 October, 2007. a question that is salient for parents of children who present clinically with Tourette syndrome: how will my child function compared with individuals in my community who do not have Tourette syndrome? Our a priori hypothesis was that individuals with Tourette syndrome in late adolescence would have lower scores on the Children's Global Assessment Scale (CGAS). ${ }^{10}$ We also explored whether psychosocial outcomes and rates of psychiatric comorbidity were associated with the severity of tic, attention-deficit hyperactivity disorder (ADHD) or obsessivecompulsive disorder (OCD) symptoms.

\section{Method}

\section{Participants}

Individuals with Tourette syndrome were recruited from 1993 to 1999 and consisted of two birth cohorts identified from all patients born in 1975 and 1980 and seen during childhood in the Yale Child Study Center Tourette syndrome and obsessivecompulsive disorder (OCD) clinic. To enable comparison between study participants and non-participants, families who did not participate in the in-person interviews could still complete the briefer telephone interview. Community controls were recruited from a list of 10000 names purchased from a telemarketing company, and selected for contact using a random number generator. Controls were individually matched with those in the case group on age, gender, geographic region (ZIP code), and twin status (singleton $v$. twin). At the time of recruitment, participants were approximately 18 years old.

No exclusion criteria were stipulated for individuals with Tourette syndrome. Potential controls were excluded only if they reported a history of tics, or obsessive-compulsive symptoms or 
tics were observed on interview. After a complete description of the study, written informed consent was obtained from adult probands and parents, and assent was obtained from probands $<18$ years of age. Families were paid for participation. The institutional review boards at Yale University School of Medicine, New York State Psychiatric Institute, Columbia University College of Physicians and Surgeons and The Hospital for Sick Children approved the study.

\section{Psychosocial outcome measures}

Our primary psychosocial outcome measure was the CGAS, an adaptation of the Global Assessment Scale (GAS) ${ }^{11}$ for youth aged 4-16 years. It is a valid and reliable instrument used widely in research and clinically to assess overall psychiatric and social functioning of youth. ${ }^{10,12}$ Children's Global Assessment Scale ratings range from 1 (lowest level of functioning) to 100 (highest). Although the mean (s.d.) age of our Tourette syndrome sample at reassessment was 18.1 (1.4) years (range 15.7-20.8), we selected the CGAS instead of a comparable scale for adults because we thought it would be superior for assessing older adolescents. Moreover, the theoretical reason for choosing 16 years as the upper age limit for the CGAS is not documented, and it has been used up to age 23 without reducing interrater reliability. ${ }^{13}$

A consensus procedure was used by two child psychiatrists (D.A.G. and K.J.P.) to determine a CGAS score for each participant. They were masked to group status so that their ratings would not be influenced by knowledge of whether the individual had Tourette syndrome. Because a history of tics or obsessivecompulsive symptoms was the only exclusion criterion for potential controls, the raters had access to all collected information that did not refer to these symptoms. They determined CGAS scores independently and then conferred to resolve discrepancies $\geqslant 10$ (the mean was used for discrepancies $<10$ ). The interrater reliability was high (intraclass correlation 0.8 ).

Secondary outcome measures included the Socialization domain of the Vineland Adaptive Behaviour Scales, ${ }^{14}$ the Child Behaviour Checklist (CBCL) ${ }^{15}$ and the Youth Self-Report (YSR). ${ }^{16}$ The Vineland is a reliable and valid measure of personal and social functioning in youth aged 6-19 years. It is administered as a semi-structured interview with a primary caregiver. The CBCL is a reliable and valid parent-report measure for assessing problem behaviour and adaptive functioning in youth aged 4-18 years. The YSR, which also has adequate reliability and validity, is closely related to the CBCL and is designed to be completed by youth aged 11-18 years.

\section{Assessment of psychiatric comorbidity}

Another consensus procedure was used by two child psychiatrists (J.F.L. and B.S.P.) to determine lifetime DSM-IV ${ }^{17}$ diagnoses for all participants. Again, the child psychiatrists were masked to group status and made determinations based on all data that did not refer to tics or obsessive-compulsive symptoms. After making diagnoses independently, they conferred to resolve differences. They also used the same procedure to confirm the diagnosis of Tourette syndrome (ignoring the impairment criterion $)^{18}$ in the individuals with Tourette syndrome.

\section{Procedures for data collection}

Demographic and clinical information was collected from the clinic chart and several other sources. The telephone interview, administered to a parent, included questions regarding the potential proband's history of tics, ADHD and obsessive-compulsive symptoms and learning. For individuals with potential Tourette syndrome, it also assessed current tic severity using the Yale Global Tic Severity Scale (YGTSS). ${ }^{19}$ The interviewer could not be masked to the group status of the potential proband because of the need to rule out a history of tics and obsessive-compulsive symptoms in potential controls, and to assess current tic severity in those with potential Tourette syndrome.

Parent and proband in-person interviews included the Revised Schedule for Tourette and Other Behavioural Syndromes (STOBS-R), a semi-structured interview used extensively in family-genetic studies. ${ }^{20}$ Parents were interviewed twice by different interviewers to prevent the assessment of tic and obsessive-compulsive symptoms during one interview from biasing the masked assessment of other symptoms and psychosocial functioning during the other. The former interview was not masked to group status and included sections of the Adulton-Child STOBS-R pertaining to tic and obsessive-compulsive symptoms. For participants with Tourette syndrome, it also included assessments of current and worst-ever tic and OCD severity using the YGTSS and the Yale-Brown Obsessive Compulsive Scale (Y-BOCS). ${ }^{21,22}$ The other interview, where a different interviewer was masked to group status, included the Vineland Socialization domain and the portion of the Adult-onChild STOBS-R that contains the Schedule for Affective Disorders and Schizophrenia for School-Age Children, Epidemiologic Version (Kiddie-SADS-E). ${ }^{23}$ In the proband interview, the Child-on-Child STOBS-R was supplemented for individuals with Tourette syndrome with the YGTSS. The interviewer could not be masked to group status for any portion of the proband interview, because a proband's tics could be observed.

In addition to the CBCL (completed by parents) and YSR (completed by the proband), self-report measures included the ADHD Rating Scale-IV-Home Version ${ }^{24}$ (completed separately by parents and the proband for current and worst-ever symptoms).

Despite our best efforts to collect all data for each participant, some families did not complete every secondary psychosocial outcome or symptom severity measure (completion rates are provided in online Table DS1).

\section{Data analysis}

Statistical analyses were performed using SPSS v.16.0 for Windows. In our primary analysis, CGAS scores were compared in the participants with Tourette syndrome and controls using analysis of covariance (ANCOVA). The groups were matched perfectly on gender, and we controlled statistically for age and socioeconomic status (quantified using the Hollingshead Four-Factor Index of Social Status). ${ }^{25}$

All other analyses were considered exploratory. The approach used for the primary analysis was also used to assess group differences in the secondary psychosocial outcome measures (Vineland Socialization domain, CBCL and YSR). Group differences in lifetime rates of psychiatric disorders were assessed with chi-squared analyses and conditional logistic regression, applying the Cox regression option in SPSS and controlling for age and socioeconomic status. Because differences in outcome between groups might be related to the influence of ADHD, ${ }^{26}$ ANCOVA and conditional logistic regression analyses were repeated with the presence or severity of ADHD entered into the models as covariates. We also assessed the influence of ADHD by using ANCOVA to compare psychosocial outcomes in individuals with Tourette syndrome with and without ADHD and the controls (controlling for age, gender and socioeconomic status).

Within the Tourette syndrome group, ANCOVA was used to assess whether psychosocial outcomes were associated with a lifetime diagnosis of ADHD or OCD, and linear regression was 
used to assess whether these outcomes were associated with the severity of tic (based on the YGTSS excluding the impairment scale), ADHD or obsessive-compulsive symptoms. Similarly, logistic regression was used to assess whether comorbid psychiatric disorders were associated with the same variables. Age, gender and socioeconomic status were entered into the models as covariates. The significance level for all analyses was $P=0.05$ (two-tailed). This was not adjusted for multiple comparisons because we had only one a priori hypothesis and the rest of the analyses were exploratory.

\section{Results}

\section{Group characteristics}

The sample comprised 65 individuals with Tourette syndrome and 65 community controls. Few potential controls were excluded on account of tics or obsessive-compulsive symptoms being discovered. Participants with Tourette syndrome received their initial clinical evaluation at a mean age of $10.8($ s.d. $=2.8)$ years and were reassessed 7.3 (s.d. $=3.1$ ) years later. Non-participants with Tourette syndrome included 1 individual who died of a cerebral malignancy, 12 who refused to participate and 2 whose reason for non-participation is unclear. Of the 14 surviving non-participants, 9 completed the telephone interview. The 65 participants with Tourette syndrome and these 9 non-participants were comparable on demographic and clinical variables (data are provided in online Table DS2). The Tourette syndrome group and controls were similar in age (mean 18.1 (s.d. =1.4) v. $18.4(1.7)$ years, $t_{(128)}=1.2, \quad P=0.2$ ) and socioeconomic status (mean Hollingshead score 49.6 (s.d.=10.8) v. $52.2(8.0), t_{(128)}=1.5$, $P=0.1$ ). Individual matching of the Tourette syndrome group with controls was perfect on gender (57 males per group) and twin status (five twins, including two twin pairs, per group). In each group, all participants but one were White. All measures of tic and OCD severity showed that current symptoms were significantly improved compared with worst-ever symptoms in the Tourette syndrome group, and the same was true for ADHD symptoms in the Tourette syndrome group and controls (current and worst-ever symptom severity data are provided in online Table DS3).

\section{Group comparisons}

Scores on the CGAS, our primary psychosocial outcome measure, were significantly and substantially lower in the Tourette syndrome group than controls, and this difference remained significant when controlling for a lifetime diagnosis of ADHD (Table 1; complete data shown in online Table DS4) or any measure of ADHD severity (current or worst-ever symptoms rated by parents or probands). Similarly, Vineland Socialization standard scores were significantly lower in the Tourette syndrome group, and this difference also remained significant when controlling for each ADHD covariate. The CGAS scores correlated positively with Vineland Socialization scores $\left(r=0.5, P=10^{-9}\right)$.

On the CBCL, participants with Tourette syndrome had lower $\mathrm{t}$-scores on all competence subscales and higher t-scores on all syndrome subscales than controls, and most of these differences were highly significant. Moreover, internalising, externalising, and total problems t-scores were significantly higher in individuals with Tourette syndrome. When we controlled for ADHD diagnosis or severity, many of the group differences on the CBCL remained significant.

On the YSR, the Tourette syndrome group and controls had similar t-scores on all syndrome subscales, except for significantly higher attention problems in those with Tourette syndrome $($ mean $=58.3$ (s.d. =9.8) v. $54.8(6.2), F=4.6(1,103), P=0.03)$. In contrast, those in the Tourette syndrome group had significantly lower t-scores on both competence subscales, activities $($ mean $=45.0$ (s.d. $=7.8)$ v. $49.6(6.3), F=13.9(1,88), P<0.001)$ and social $($ mean $=46.8$ (s.d. $=8.1)$ v. $51.1(6.9), F=6.6(1,84)$, $P=0.01$ ). Differences on the attention problems subscale and the social competence subscale were no longer significant, however, when we controlled for ADHD diagnosis or parent-rated ADHD severity (current or worst-ever). No significant differences were found in internalising, externalising or total problems t-scores.

Compared with controls and participants with Tourette syndrome but not ADHD, those with Tourette syndrome and ADHD had significantly lower scores on the CGAS and Vineland Socialization domain, and significantly higher t-scores on the CBCL internalising, externalising, and total problems scales and the YSR externalising scale. In contrast, those in the Tourette

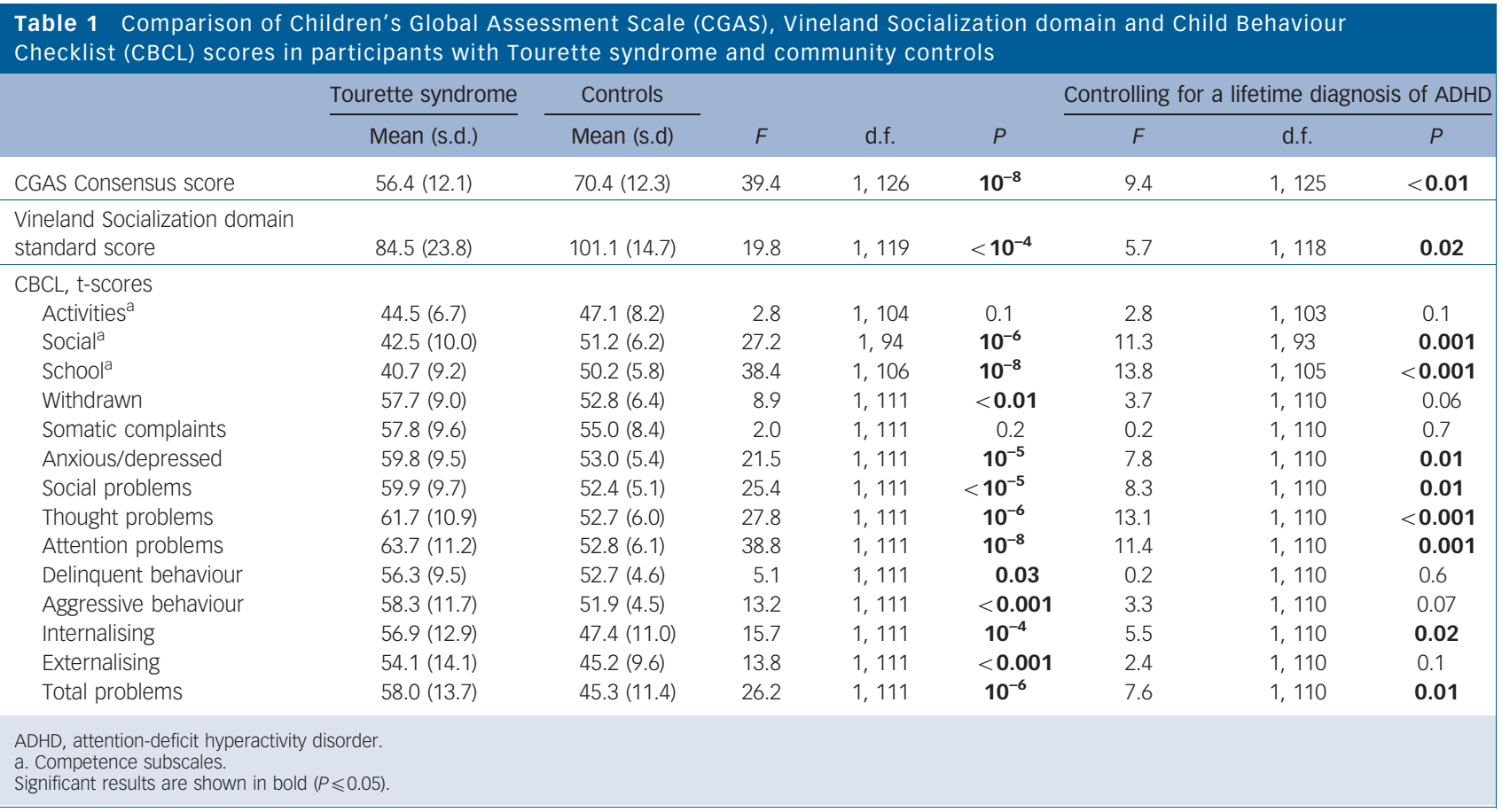


Table 2 Comparison of lifetime psychiatric disorders in the Tourette syndrome group and community controls

\begin{tabular}{|c|c|c|c|c|c|c|c|c|}
\hline & \multirow{3}{*}{$\begin{array}{c}\text { Tourette } \\
\text { syndrome } \\
(n=65) \\
n(\%)\end{array}$} & \multirow{3}{*}{$\begin{array}{c}\text { Controls } \\
(n=65) \\
n(\%)\end{array}$} & \multirow{2}{*}{\multicolumn{2}{|c|}{ Test statistic }} & \multirow[b]{3}{*}{ OR $(95 \% \mathrm{Cl})$} & \multicolumn{3}{|c|}{ Conditional logistic regression } \\
\hline & & & & & & \multirow[b]{2}{*}{$P$} & \multicolumn{2}{|c|}{ Controlling for a lifetime diagnosis of $A D H D$} \\
\hline & & & $\chi^{2}($ d.f. $=1)$ & $P$ & & & OR $(95 \% \mathrm{Cl})$ & $P$ \\
\hline \multicolumn{9}{|l|}{$\begin{array}{l}\text { Any psychiatric disorder } \\
\text { (including } O C D)^{a, b}\end{array}$} \\
\hline $\begin{array}{l}\text { Any psychiatric disorder } \\
\text { except OCD }\end{array}$ & \multicolumn{7}{|c|}{ Any psychiatric disorder } & 0.04 \\
\hline ADHD & $43(66.2)$ & $9(13.8)$ & 37.1 & $10^{-9}$ & $7.3(2.8-19.5)$ & $10^{-4}$ & - & - \\
\hline$O C D^{b}$ & $25(38.5)$ & - & - & - & - & - & - & - \\
\hline & 0.3 \\
\hline Learning disorder $^{\mathrm{d}}$ & $27(41.5)$ & $8(12.3)$ & 14.1 & $<0.001$ & $7.9(2.2-28.2)$ & 0.001 & $3.5(0.9-14.4)$ & 0.08 \\
\hline Stuttering & $8(12.3)$ & $5(7.7)$ & 0.8 & 0.4 & $2.2(0.5-9.7)$ & 0.3 & $3.0(0.5-17.4)$ & 0.2 \\
\hline Conduct disorder & $15(23.1)$ & $2(3.1)$ & 11.4 & 0.001 & $7.8(1.7-36.8)$ & 0.01 & $3.7(0.7-21.5)$ & 0.1 \\
\hline Major depressive disorder & $40(61.5)$ & $17(26.2)$ & 16.5 & $10^{-4}$ & $4.2(1.8-9.7)$ & 0.001 & $3.6(1.4-9.2)$ & 0.01 \\
\hline Dysthymic disorder & $7(10.8)$ & $3(4.6)$ & 1.7 & 0.2 & $3.8(0.9-16.7)$ & 0.08 & $3.0(0.5-19.0)$ & 0.2 \\
\hline Bipolar disorder & $4(6.2)$ & $0(0.0)$ & $2.3^{g}$ & 0.1 & - & - & - & - \\
\hline $\begin{array}{l}\text { Primary psychotic } \\
\text { disorder }^{\mathrm{e}}\end{array}$ & $5(7.7)$ & $0(0.0)$ & $3.3^{g}$ & 0.07 & - & - & - & - \\
\hline Substance use disorder ${ }^{f}$ & $9(13.8)$ & $6(9.2)$ & 0.7 & 0.4 & $1.7(0.5-6.1)$ & 0.4 & $0.7(0.1-3.8)$ & 0.7 \\
\hline \multicolumn{9}{|c|}{$\begin{array}{l}\text { OCD, obsessive-compulsive disorder; ADHD, attention-deficit hyperactivity disorder. } \\
\text { a. Includes stuttering, learning disorder (math, reading, written expression, and not otherwise specified (NOS)), developmental coordination disorder, phonological disorder, ADHD, } \\
\text { conduct disorder, OOD, panic disorder, specific phobias, generalised anxiety disorder, separation anxiety disorder, anxiety disorder NOS, major depression, dysthymic disorder, } \\
\text { bipolar disorder, schizophrenia, brief psychotic disorder, psychotic disorder NOS, substance misuse, and substance-induced mood disorder. } \\
\text { b. Data not provided for community controls because a history of obsessive-compulsive symptoms was an exclusion criterion for them. } \\
\text { C. Includes panic disorder, specific phobias, generalised anxiety disordes, separation anxiety disorder, and anxiety disorder NOS. } \\
\text { d. Includes learning disorder in maths, reading, and written expression and learning disorder NOS. } \\
\text { e. Includes schizophrenia, brief psychotic disorder and psychotic disorder NOS; however, all five participants with Tourette syndrome with a primary psychotic disorder diagnosis } \\
\text { had psychotic disorder NOS. } \\
\text { f. Includes alcohol, cannabis, amphetamine, cocaine, sedative/hypnotic/anxiolytic, opioid, hallucinogen, phencyclidine and other substance misuse. } \\
\text { g. With continuity correction. } \\
\text { Significant results are shown in bold ( } P \leqslant 0.05 \text { ). }\end{array}$} \\
\hline
\end{tabular}

syndrome group without ADHD and the controls did not differ significantly from each other on these measures, although they did differ significantly on several CBCL subscales (online Table DS5). These results suggest that the poorer psychosocial outcomes in the Tourette syndrome group were largely attributable to comorbid ADHD rather than Tourette syndrome itself; however, they are based on analyses that have lower statistical power than those presented in online Table DS4, which indicate that even after we controlled for the influence of ADHD, participants with Tourette syndrome still had impaired functioning compared with controls.

The Tourette syndrome group had significantly higher lifetime rates than controls of $\mathrm{ADHD}$, learning disorder, conduct disorder and major depression, and tended to have higher rates of non-OCD anxiety disorders (Table 2). When we controlled, however, for a lifetime diagnosis of ADHD or parent-rated ADHD severity (current or worst-ever), group differences in rates of learning disorder and conduct disorder were no longer significant.

\section{Within-group analyses for the Tourette syndrome group}

Within the Tourette syndrome group, associations of psychosocial outcomes with a lifetime diagnosis of ADHD and OCD and with tic, ADHD and obsessive-compulsive symptom severity are presented in Tables 3 and 4, and associations of comorbid psychiatric disorders with these variables are presented in Tables 5 and 6. Overall, worse psychosocial outcomes were associated with greater severity of tic, ADHD and obsessive-compulsive symptoms, particularly as rated by parents. Fewer associations were found between lifetime rates of psychiatric disorders and these measures. No symptom severity measure correlated with major depressive disorder.

\section{Discussion}

The major finding of this study, confirming our a priori hypothesis, is that the global functioning of older adolescents with Tourette syndrome who were identified in childhood was substantially poorer than that of community controls. Furthermore, their socialisation, according to the Vineland, was worse; they had more internalising and externalising problems according to the CBCL; and they had higher lifetime rates of other psychiatric disorders. Much of the impairment in individuals with Tourette syndrome, however, was attributable to comorbid ADHD.

Several features of the study strengthen the validity of these findings. First, the parent in-person interview was divided into two parts, with the interviewer masked to group status during the portion that included the Vineland and Kiddie-SADS-E. Second, our Tourette syndrome sample is representative of the target population, as we recruited $>80 \%$ of eligible individuals to participate and determined that non-participants were similar to participants on demographic and clinical measures. Third is the narrow age range of participants, as studies of psychiatric comorbidity based on lifetime prevalence in mixed-age samples can create the appearance of comorbidity even when disorders are randomly associated. ${ }^{27}$ Finally, we compared participants with Tourette syndrome with community controls who were matched on demographic variables and ascertained with few exclusion criteria; therefore, differences in outcome are unlikely to be related to confounding factors or to selection of controls who are particularly high functioning. Indeed, the mean CGAS score in the 

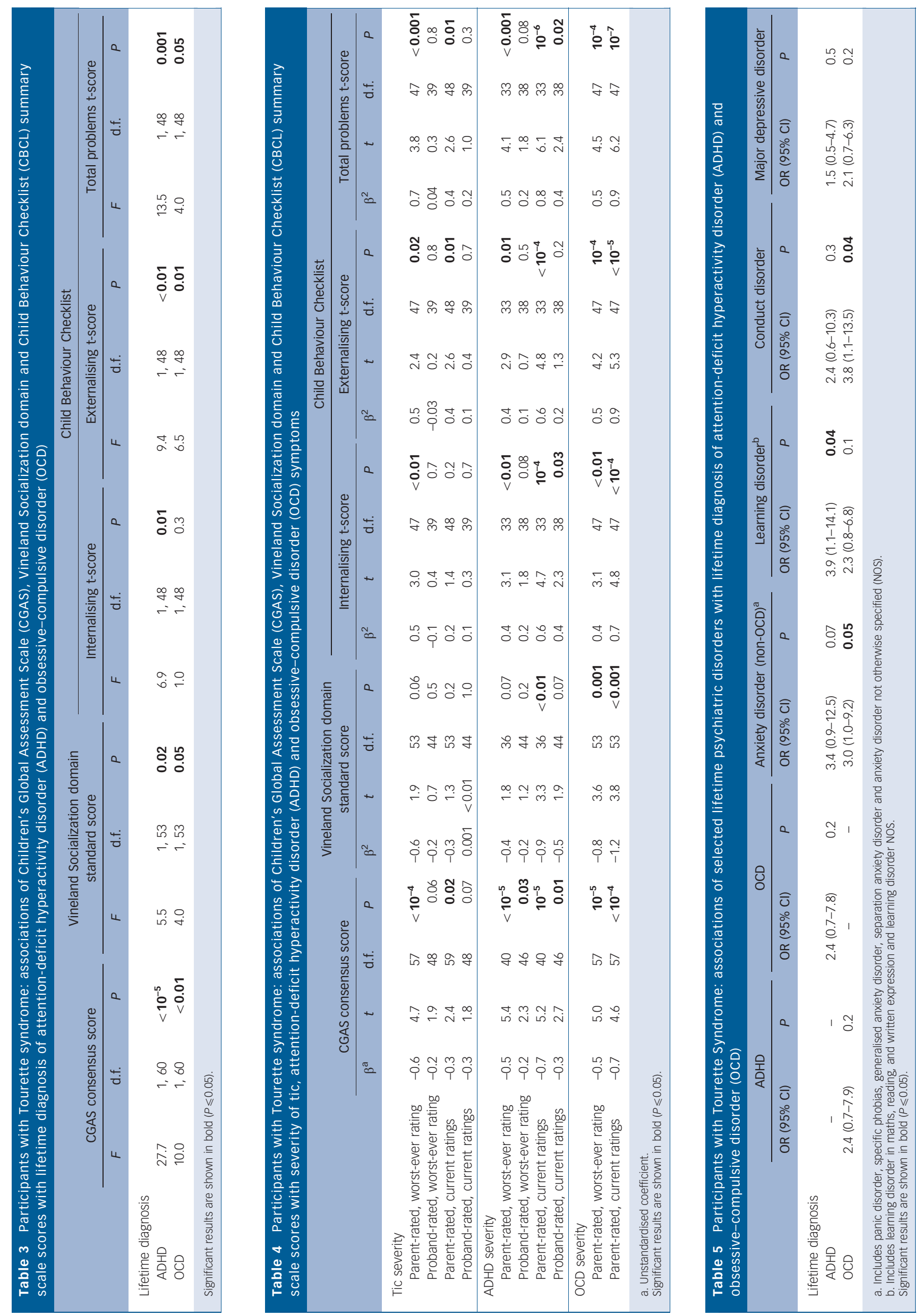


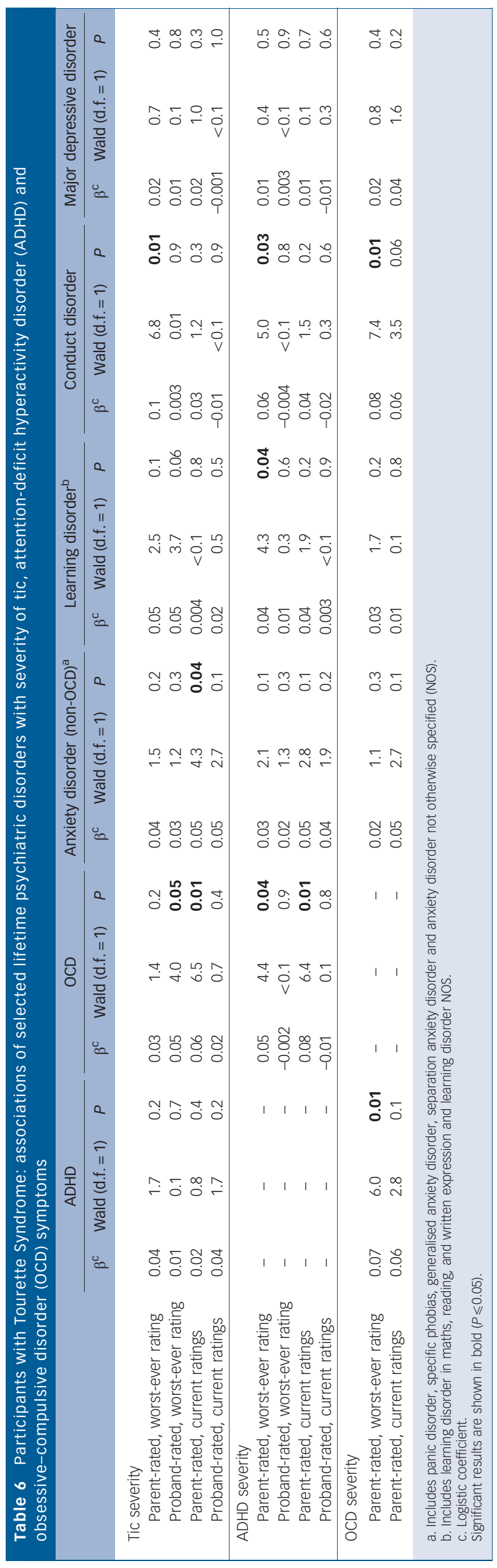

control group was right at the cut-off point for normal function ( $>70$ ) suggested by the instrument's authors. ${ }^{10}$

Our finding of impaired global functioning in individuals with Tourette syndrome reassessed in late adolescence counters results of longitudinal studies that found relatively high functional status around this age. ${ }^{5-7}$ Those studies were limited, however, by the absence of a control group, and rates of comorbid ADHD were much lower than in our sample. Our study also extends results of controlled cross-sectional studies in which children or adults with Tourette syndrome were found to have worse functioning than controls, particularly if they had comorbid ADHD, ${ }^{1,8,26}$ and higher rates of comorbid psychopathology. ${ }^{9,28}$ Furthermore, whereas previous studies of adults with Tourette syndrome recruited individuals from patients attending a specialty clinic, ${ }^{8,9}$ the Tourette syndrome group in our study was identified in childhood and then contacted for reassessment years later. As a result, we can be confident that our Tourette syndrome group does not represent a subgroup of individuals with Tourette syndrome with particularly severe or persistent tics for which they were pursuing treatment. On the contrary, their tics at the time of reassessment were generally mild.

\section{Associations of outcomes with tic severity}

The relationship between tic severity and psychosocial functioning in people with Tourette syndrome remains unclear, despite multiple attempts to examine it. Many cross-sectional reports on youth indicate that those with more severe tics have worse functioning, ${ }^{29,30}$ but many others indicate no association. ${ }^{1,26,28,31}$ The variability in findings is likely related to the wide age range of the samples, the complicated trajectory of tics during childhood, ${ }^{3}$ the large fluctuation in tic severity at any given age, and variable statistical power to detect real effects. In keeping with limited longitudinal data, ${ }^{5,6}$ however, we found that lower CGAS scores in late adolescence, determined by evaluators masked to tic history, were associated with greater tic severity as rated by parents. Similarly, higher CBCL internalising, externalising, and total problems scores were associated with more severe parentrated tics. In contrast, we found few associations in older adolescents between lifetime rates of comorbid illnesses and measures of tic severity. A notable exception was that a lifetime diagnosis of OCD was associated with more severe parent-rated current tics, suggesting that children with Tourette syndrome and comorbid OCD may be more likely to have more severe tic symptoms in late adolescence.

\section{Associations of outcomes with ADHD}

With few exceptions, ${ }^{29}$ studies of youth with Tourette syndrome indicate that comorbid ADHD is strongly associated with poorer psychosocial outcomes, ${ }^{1,26,31,32}$ and our findings support this conclusion. Nevertheless, after we adjusted for the influence of ADHD, we still found that older adolescents with Tourette syndrome had impaired functioning compared with controls. These results are consistent with a recent cross-sectional study of adults with Tourette syndrome, which found that those with a history of ADHD had significantly more psychopathology and maladaptive behaviours, but those without ADHD still had higher levels of psychopathology compared with population norms. ${ }^{33}$ Limited data regarding the association of ADHD with other comorbid disorders in individuals with Tourette syndrome have yielded mixed results, ${ }^{26,32}$ but within-group analyses in our Tourette syndrome sample indicate that parent-rated worst-ever ADHD severity was associated with OCD, learning disorder and conduct disorder, although not major depressive disorder. 
Moreover, between-group analyses reveal that ADHD diagnosis and severity accounted for elevated rates in those with Tourette syndrome of learning disorder and conduct disorder, but again not major depressive disorder. We cannot conclude from our data whether an inherent, neurobiological relationship exists between $\mathrm{ADHD}$ and the other disorders associated with it, or whether the influence of ADHD on psychosocial functioning increases the risk that they will develop.

\section{Associations of outcomes with OCD}

Some ${ }^{1,34}$ but not all ${ }^{29,31}$ studies indicate that comorbid OCD is associated with poorer psychosocial functioning in youth with Tourette syndrome. The inconsistency likely stems from the young and variable ages of the samples in relation to the usual adolescent age at onset of OCD in the context of pre-existing Tourette syndrome. ${ }^{6}$ We found, however, that not only current but also worst-ever OCD severity was associated with poorer functioning in late adolescence according to the CGAS, Vineland and CBCL, suggesting that OCD in childhood can have long-term psychosocial repercussions. We also found that a history of OCD was associated with higher lifetime rates of conduct disorder and non-OCD anxiety disorders, and patients with more severe obsessive-compulsive symptoms were more likely to have conduct disorder and ADHD. Although a previous study of adults with Tourette syndrome found no meaningful differences between those with and without comorbid OCD in rates of other comorbid disorders, ${ }^{35}$ that study had a smaller sample, did not assess for conduct disorder and did not report associations with OCD severity. Moreover, our results are consistent with a previous report that OCD was associated with higher rates of other anxiety disorders in youth with Tourette syndrome, ${ }^{36}$ and with epidemiologically based findings that OCD was associated with prior separation anxiety disorder, ADHD and conduct problems. ${ }^{37}$

\section{Relationship of Tourette syndrome with major depression}

Our finding that nearly two-thirds of older adolescents with Tourette syndrome had a lifetime diagnosis of major depressive disorder is consistent with many previous reports indicating a strong association between Tourette syndrome and depression in clinical samples. ${ }^{38}$ The reason for this association, however, remains a source of controversy. Some maintain that depression is an integral part of Tourette syndrome and shares a common genetic basis, ${ }^{39}$ whereas others argue that the disorders are genetically unrelated, and that Tourette syndrome is a risk factor for depression because of the emotional consequences of having a chronic illness. ${ }^{40}$ In keeping with the latter hypothesis, the influence of tic severity, ADHD, OCD and psychosocial stress have been thought to account for the association of Tourette syndrome with depression. ${ }^{34,38}$ Our data, however, argue against this possibility, as within the Tourette syndrome group, major depressive disorder was the only comorbid disorder for which we found no association with a lifetime diagnosis of ADHD or OCD, or any measure of tic, ADHD or OCD severity. Moreover, unlike learning disorder and conduct disorder, the between-group difference in rates of major depressive disorder remained significant after controlling for ADHD diagnosis or severity. The association of Tourette syndrome with depression requires further investigation, but our data suggest that these disorders could be inherently linked, independent of the burden of chronic illness.

\section{Limitations}

As in most studies of Tourette syndrome, we recruited individuals from a specialty clinic, and therefore the psychosocial impairment and high rates of comorbidity we found may be partly related to Berkson's bias. ${ }^{41}$ In addition, the findings may not be generalisable to all individuals with Tourette syndrome, especially those who do not present clinically and receive a diagnosis. Nevertheless, it is precisely the clinical nature of our sample that makes our findings clinically relevant, as they pertain to children whose parents are sufficiently concerned about their child's tic symptoms to pursue a clinical assessment. A sample of epidemiologically ascertained youth would include many whose symptoms were unrecognised or of little or no concern. Furthermore, although our Tourette syndrome group was recruited from a specialty clinic in a single tertiary care centre, most received their initial diagnostic assessment for Tourette syndrome at that same centre, and very few were referred to us because previously diagnosed Tourette syndrome was too challenging to be managed elsewhere. Consequently, our sample is likely similar to that of individuals who receive a diagnosis of Tourette syndrome in less specialised clinical settings. Most of our participants were middle-class and White, however, and therefore our findings may not generalise to other ethnic and socioeconomic groups. On the other hand, using a relatively privileged sample such as ours is likely to bias the results in favour of better outcomes, and even in our sample we found that older adolescents with Tourette syndrome experienced considerable morbidity.

The CGAS scores were determined retrospectively, and although the raters followed the instrument's guidelines, the exact scores assigned inevitably depended on the subjective judgement of the raters. Retrospective rating of the CGAS was necessary, however, to ensure that the raters were masked to group status. Furthermore, the CGAS's interrater and test-retest reliability, as well as its concurrent and discriminant validity, have been amply demonstrated..$^{10,12,13}$ In our study, the interrater reliability of CGAS scores was likewise high, and their validity was supported by their significant correlation with Vineland Socialization scores. The assessments of worst-ever symptom severity were also retrospective and therefore could have been influenced by recall bias. Despite this possibility, it is reassuring that in the Tourette syndrome group the associations of psychosocial outcomes with parent-rated worst-ever ADHD and OCD severity were generally consistent with the associations of these outcomes with a lifetime diagnosis of ADHD and OCD (determined by two child psychiatrists and based on multiple sources of information).

The naturalistic design of our study did not permit an analysis of treatment effects, although $85 \%$ of participants with Tourette syndrome were treated pharmacologically at some time during the course of their illness. This treatment history is consistent with the psychosocial impairment that our participants experienced, and it suggests that the long-term benefits of pharmacotherapy may be limited for individuals with such complex psychopathology. Although we found that individuals with Tourette syndrome had impaired psychosocial functioning as older adolescents, assessment in later years is necessary to determine whether this impairment persists into adulthood.

In the interest of generating hypotheses and minimising the risk of Type II errors, we conducted multiple exploratory analyses without adjusting the significance level of $P=0.05$. Therefore, we cannot rule out the possibility that some post hoc findings may have occurred by chance. However, even when we applied the more stringent significance level of $P=0.001$ the overall pattern of results was largely unchanged.

\section{Implications}

Our study provides new information to help clinicians counsel parents whose children have Tourette syndrome. Previous reports indicate that children with Tourette syndrome generally have 
impaired psychosocial functioning that is attributable in large part to ADHD and possibly OCD and tic severity. ${ }^{1,26,29,30}$ The prognosis for psychosocial functioning, however, has been unclear. Our data show that older adolescents with Tourette syndrome who were identified in childhood continue to experience considerable psychosocial impairment, and they have high lifetime rates of $\mathrm{ADHD}$, major depressive disorder, learning disorder, OCD, non-OCD anxiety disorders and conduct disorder. Thus, although parents can be reassured that tics are likely to improve by 18 years of age, ${ }^{3,4}$ outcomes for psychosocial functioning and comorbid conditions are generally less optimistic. Our study also offers clinical clues to inform the prognosis for an individual child with Tourette syndrome. Poorer outcomes in late adolescence are associated not only with more severe ADHD symptoms in childhood, but also with more severe OCD symptoms and tics. In contrast, although the risk of developing major depressive disorder is high, it appears to be independent of the severity of these symptoms. Overall, our findings highlight the importance of closely monitoring children with Tourette syndrome as they mature for psychosocial impairment and comorbid conditions.

Daniel A. Gorman, MD, FRCPC, The Hospital for Sick Children and Department of Psychiatry, University of Toronto, Canada; Nancy Thompson, BA, Yale University Child Study Center, New Haven, Connecticut, USA; Kerstin J. Plessen, MD, PhD Department of Biological and Medical Psychology, Cognitive Neuroscience Group, Department of Biological and M, Concal Psych, University of Bergen, Bergen, Norway; Mary M. Robertson, MBChB, MD, DSC (Med) DPM, FRCPCH, FRCP (UK), FRCPsych, Department of Mental Health Sciences, University College London, London; James F. Leckman, MD, Yale University Child Study Center and Departments of Pediatrics, Psychiatry and Psychology, New Haven Connecticut, USA; Bradley S. Peterson, MD, Department of Psychiatry, Columbia University College of Physicians and Surgeons and the New York State Psychiatric Institute, New York, USA

Correspondence: Daniel A. Gorman, MD, FRCPC, The Hospital for Sick Children, 555 University Avenue, Toronto M5G 1X8, Canada. Email: daniel.gorman@sickkids.ca

First received 22 Jul 2009, final revision 1 Feb 2010, accepted 11 Feb 2010

\section{Funding}

This work was supported in part by a grant from the March of Dimes (BSP); National Institutes of Health grant K02-74677 (BSP); and the Suzanne Crosby Murphy Endowmen at Columbia University College of Physicians and Surgeons.

\section{Acknowledgements}

The authors thank Hongtu Zhu, PhD, for providing statistical consultation.

\section{References}

1 Carter AS, O'Donnell DA, Schultz RT, Scahill L, Leckman JF, Pauls DL. Social and emotional adjustment in children affected with Gilles de la Tourette's syndrome: associations with ADHD and family functioning. J Child Psychol Psychiatry 2000; 41: 215-23.

2 Kurlan R, Como PG, Miller B, Palumbo D, Deeley C, Andresen EM, et al. The behavioural spectrum of tic disorders: a community-based study. Neurology 2002; 59: 414-20.

3 Leckman JF, Zhang H, Vitale A, Lahnin F, Lynch K, Bondi C, et al. Course of tic severity in Tourette's syndrome: the first two decades. Pediatrics 1998; 102: 14-9.

4 Pappert EJ, Goetz CG, Louis ED, Blasucci L, Leurgans S. Objective assessments of longitudinal outcome in Gilles de la Tourette's syndrome. Neurology 2003; 61: 936-40.

5 Leckman JF, Bloch MH, King RA, Scahill L. Phenomenology of tics and natural history of tic disorders. Adv Neurol 2006; 99: 1-16.

6 Bloch $\mathrm{MH}$, Peterson BS, Scahill L, Otka J, Katsovich L, Zhang $\mathrm{H}$, et al. Adulthood outcome of tic and obsessive-compulsive symptom severity in children with Tourette syndrome. Arch Pediatr Adolesc Med 2006; 160: 65-9.
7 Burd L, Kerbeshian PJ, Barth A, Klug MG, Avery PK, Benz B. Long-term follow-up of an epidemiologically defined cohort of patients with Tourette syndrome. J Child Neurol 2001; 16: 431-7.

8 Elstner K, Selai CE, Trimble MR, Robertson MM. Quality of Life (QOL) of patients with Gilles de la Tourette's syndrome. Acta Psychiatr Scand 2001; 103: 52-9.

9 Robertson MM, Banerjee S, Hiley PJ, Tannock C. Personality disorder and psychopathology in Tourette's syndrome: a controlled study. Br J Psychiatry 1997; 171: 283-6.

10 Shaffer D, Gould MS, Brasic J, Ambrosini P, Fisher P, Bird H, et al. A children's global assessment scale (CGAS). Arch Gen Psychiatry 1983; 40: 1228-31.

11 Endicott J, Spitzer RL, Fleiss JL, Cohen J. The global assessment scale: a procedure for measuring overall severity of psychiatric disturbance. Arch Gen Psychiatry 1976; 33: 766-71.

12 Green B, Shirk S, Hanze D, Wanstrath J. The Children's Global Assessment Scale in clinical practice: an empirical evaluation. J Am Acad Child Adolesc Psychiatry 1994; 33: 1158-64.

13 Schorre BE, Vandvik IH. Global assessment of psychosocial functioning in child and adolescent psychiatry: a review of three unidimensional scales (CGAS, GAF, GAPD). Eur Child Adolesc Psychiatry 2004; 13: 273-86.

14 Sparrow SS, Balla DA, Cicchetti DV. Vineland Adaptive Behaviour Scales. American Guidance Service, 1984.

15 Achenbach TM. Manual for the Child Behaviour Checklist/4-18 and 1991 Profile. University of Vermont Press, 1991.

16 Achenbach TM. Manual for the Youth Self-Report and 1991 Profile. University of Vermont, Department of Psychiatry, 1991.

17 American Psychiatric Association. Diagnostic and Statistical Manual of Mental Disorders (4th edn) (DSM-IV). APA, 1994.

18 Freeman RD, Fast DK, Burd L, Kerbeshian J, Robertson MM, Sandor P. An international perspective on Tourette syndrome: selected findings from 3,500 individuals in 22 countries. Dev Med Child Neurol 2000; 42: 436-47.

19 Leckman JF, Riddle MA, Hardin MT, Ort SI, Swartz KL, Stevenson J, et al. The Yale Global Tic Severity Scale: initial testing of a clinician-rated scale of tic severity. J Am Acad Child Adolesc Psychiatry 1989; 28: 566-73.

20 Pauls DL, Hurst CR. Schedule for Tourette and Other Behavioural Syndromes. Yale University Child Study Center, 1996.

21 Goodman WK, Price LH, Rasmusson SA, Mazure C, Delgado P, Henninger GR, et al. The Yale-Brown Obsessive Compulsive Scale: I. Development, use, and reliability. Arch Gen Psychiatry 1989; 46: 1006-11.

22 Goodman WK, Price LH, Rasmusson SA, Mazure C, Delgado P, Henninger GR, et al. The Yale-Brown Obsessive Compulsive Scale: II. Validity. Arch Gen Psychiatry 1989; 46: 1012-8.

23 Orvaschel H, Puig-Antich J. Schedule for Affective Disorders and Schizophrenia for School-Age Children-Epidemiologic, 4th Version. Nova University, Center for Psychological Study, 1987.

24 Dupaul GJ, Power TJ, Anastopoulos AD, Reid R. ADHD Rating Scale-IV: Checklists, Norms, and Clinical Interpretation. Guilford Press, 1998.

25 Hollingshead AB. Four Factor Index of Social Status. Yale University Press, 1975 .

26 Sukhodolsky DG, Scahill L, Zhang H, Peterson BS, King RA, Lombroso PJ, et al. Disruptive behaviour in children with Tourette's syndrome: association with ADHD comorbidity, tic severity, and functional impairment. J Am Acad Child Adolesc Psychiatry 2003; 42: 98-105.

27 Kraemer $\mathrm{HC}$, Wilson KA, Hayward C. Lifetime prevalence and pseudocomorbidity in psychiatric research. Arch Gen Psychiatry 2006; 63: $604-8$.

28 Khalifa N, von Knorring AL. Psychopathology in a Swedish population of school children with tic disorders. J Am Acad Child Adolesc Psychiatry 2006; 45: 1346-53.

29 Coffey BJ, Biederman J, Geller DA, Spencer TJ, Kim GS, Bellordre CA, et al. Distinguishing illness severity from tic severity in children and adolescents with Tourette's disorder. J Am Acad Child Adolesc Psychiatry 2000; 39 : $556-61$

30 Storch EA, Merlo LJ, Lack C, Milsom VA, Geffken GR, Goodman WK, et al. Quality of life in youth with Tourette's syndrome and chronic tic disorder. J Clin Child Adolesc Psychol 2007; 36: 217-27.

31 Hoekstra PJ, Steenhuis MP, Troost PW, Korf J, Kallenberg CG, Minderaa RB. Relative contribution of attention-deficit hyperactivity disorder, obsessivecompulsive disorder, and tic severity to social and behavioural problems in tic disorders. J Dev Behav Pediatr 2004; 25: 272-9.

32 Spencer $\mathrm{T}$, Biederman J, Harding M, O'Donnell $\mathrm{D}$, Wilens $\mathrm{T}$, Faraone $\mathrm{S}$, et al. Disentangling the overlap between Tourette's disorder and ADHD. J Child Psychol Psychiatry 1998; 39: 1037-44 
33 Haddad ADM, Umoh G, Bhatia V, Robertson MM. Adults with Tourette's syndrome with and without attention deficit hyperactivity disorder. Acta Psychiatr Scand 2009; 120: 299-307.

34 Lin $\mathrm{H}$, Katsovich $\mathrm{L}$, Ghebremichael $\mathrm{M}$, Findley $\mathrm{DB}$, Grantz $\mathrm{H}$, Lombroso $\mathrm{P}$ et al. Psychosocial stress predicts future symptom severities in children and adolescents with Tourette syndrome and/or obsessive-compulsive disorder. J Child Psychol Psychiatry 2007; 48: 157-66.

35 Coffey BJ, Miguel EC, Biederman J, Baer L, Rauch SL, O'Sullivan RL, et al. Tourette's disorder with and without obsessive-compulsive disorder in adults: are they different? J Nerv Ment Dis 1998; 186: 201-6.

36 Coffey BJ, Biederman J, Smoller JW, Geller DA, Sarin P, Schwartz S, et al. Anxiety disorders and tic severity in juveniles with Tourette's disorder. J Am Acad Child Adolesc Psychiatry 2000; 39: 562-8.

37 Peterson BS, Pine DS, Cohen P, Brook J. A prospective, longitudinal study of tic, obsessive-compulsive, and attention-deficit/hyperactivity disorders in an epidemiological sample. J Am Acad Child Adolesc Psychiatry 2001; 40: $685-95$

38 Robertson MM. Mood disorders and Gilles de la Tourette's syndrome: An update on prevalence, etiology, comorbidity, clinical associations, and implications. J Psychosom Res 2006; 61: 349-58.

39 Comings DE, Comings BG. A controlled family history study of Tourette's syndrome: III. Affective and other disorders. J Clin Psychiatry 1990; 51 288-91.

40 Pauls DL, Leckman JF, Cohen DJ. Evidence against a genetic relationship between Tourette's syndrome and anxiety, depression, panic and phobic disorders. Arch Gen Psychiatry 1994; 164: 215-21.

41 Berkson JB. Limitations of the application of fourfold table analysis to hospital data. Biometrics 1946; 2: 47-51.

\section{psychiatry in the movies}

\section{Oedipus wrecked}

\section{Peter Byrne}

The legend, tragic play, psychoanalytic theory and film plot line are tunefully summarised by Howard Dietz's lyrics to that Hollywood perennial That's Entertainment:

where a man kills his fadda

and causes a lot of bother

No film cited below offers any more sophisticated an analysis of the Oedipus complex than that song. People make films for the money and/or the attention: the trick is the pretence of High Art.

The villain of Strangers on a Train (1951), Bruno, attempts to persuade unhappily married Guy to kill Bruno's father; in return, he makes Guy a widower. Reviewers describe Bruno as a 'psychotic mother's boy'. Jimmy Piersal's biopic Fear Strikes Out (1957) portrays his depression as somehow caused by his over-competitive father. Similarly, Shine (1996) explains David Helfgott's psychotic breakdown in terms of a brutal father (traumatised by Nazi concentration camps) and an unhappy family. Helfgott's real life siblings were even more unhappy with their father's misrepresentation. Another ungrateful son, in Affliction (1997), looks like he is turning into his father, so he kills him. Patricide sets up the action of Gladiator (2000). Even comedy psychiatrists drag the ghost of Oedipus into their psychobabble: Analyse This (1999) and Analyse That (2002). Beyond the psychiatry film genre we have Westerns as the ultimate Oedipal quests. Fathers are either weak (Shane, 1953 and 3:10 to Yuma, 1957, 2007) or absent (The Cowboys, 1972). Without these familiar motifs, we would not have had what Leslie Halliwell deemed the juvenilisation of cinema: Return of the Jedi (1983).

When Niles takes over his brother's radio phone-in show in television's Frasier, he explains that he (unlike his older brother) is a Jungian: 'so there'll be no more blaming mother'. Cody Jarrett (James Cagney) in White Heat (1949) becomes a sadistic criminal not because he is a bad person, but because of a domineering mother. We are told he has 'a fierce psychopathic devotion to his mother', and to gain his trust, any new friend will have to 'take mother's place'. Bad mothers do not just make violent sons in cinema they also cause psychosis (Normal Bates in Psycho (1959), its sequels, remakes and countless imitators). In The Miracle (1991), Jimmy almost does what Freud thought he might have wanted, and in Braindead (1992), mother's boy Lionel is pulled back into mother's womb; and then there is Spanking the Monkey (1994). By Spider (2002), we are back in familiar territory. Over-attachment to mother causes psychosis. And psychosis plus cinema equals murder. 